\title{
An investigation of shock strengthening in a conical convergent channel
}

\author{
By ROBERT E. SETCHELL, ERIK STORM \\ AND BRADFORD STURTEVANT
}

Graduate Aeronautical Laboratories, California Institute of Technology

(Received 18 July 1972)

The behaviour of an initially plane, strong shock wave propagating into a conical convergence is investigated experimentally and theoretically. In the experiment a $10^{\circ}$ half-angle cone is mounted on the end of a pressure-driven shock tube. Shock waves with initial Mach numbers varying from $6 \cdot 0$ to $10 \cdot 2$ are generated in argon at a pressure of 1.5 Torr. During each run local shock velocities at several positions along the cone axis are measured using a thin multi-crystal piezoelectric probe inserted from the vertex. This technique produces accurate velocity data for both the incident and reflected shock waves. In the corresponding analysis, a simplified characteristics method is used to obtain an approximate solution of the axisymmetrie diffraction equations derived by Whitham (1959).

Both the shock velocity measurements and the axisymmetric diffraction solution confirm that the incident shock behaviour is dominated by cyclic diffraction processes which originate at the entrance of the cone. Each diffraction cycle is characterized by Mach reflexion on the cone wall followed by Mach reflexion on the axis. These cycles evidently persist until the shock reaches the cone vertex, where the measured velocity has increased by as much as a factor of three. Realgas effects, enhanced in the experiment by increasing the initial Mach number and decreasing the pressure, apparently alter the shock wave behaviour only in the region near the vertex. Velocity measurements for the reflected shock within the cone show that the shock velocity is nearly constant throughout most of the convergence length.

\section{Introduction}

A shock wave propagating into a gradually converging channel experiences a progressive strengthening. This behaviour is illustrated in figure 1, which depicts both the incident and reflected shock trajectories in a closed conical convergence. The earliest analysis of such a shock motion is the similarity solution of Guderley (1942) for converging cylindrical or spherical shock waves. This solution, later reworked with improved accuracy by Butler (1954), predicts a power-law increase in Mach number as the shock approaches the axis or point of symmetry. At the instant of shock collapse the solution is singular, but the subsequent reflected shock motion again follows a simple power law. Chisnell (1957) extended the linearized analysis of Chester (1954) to obtain an approximate 

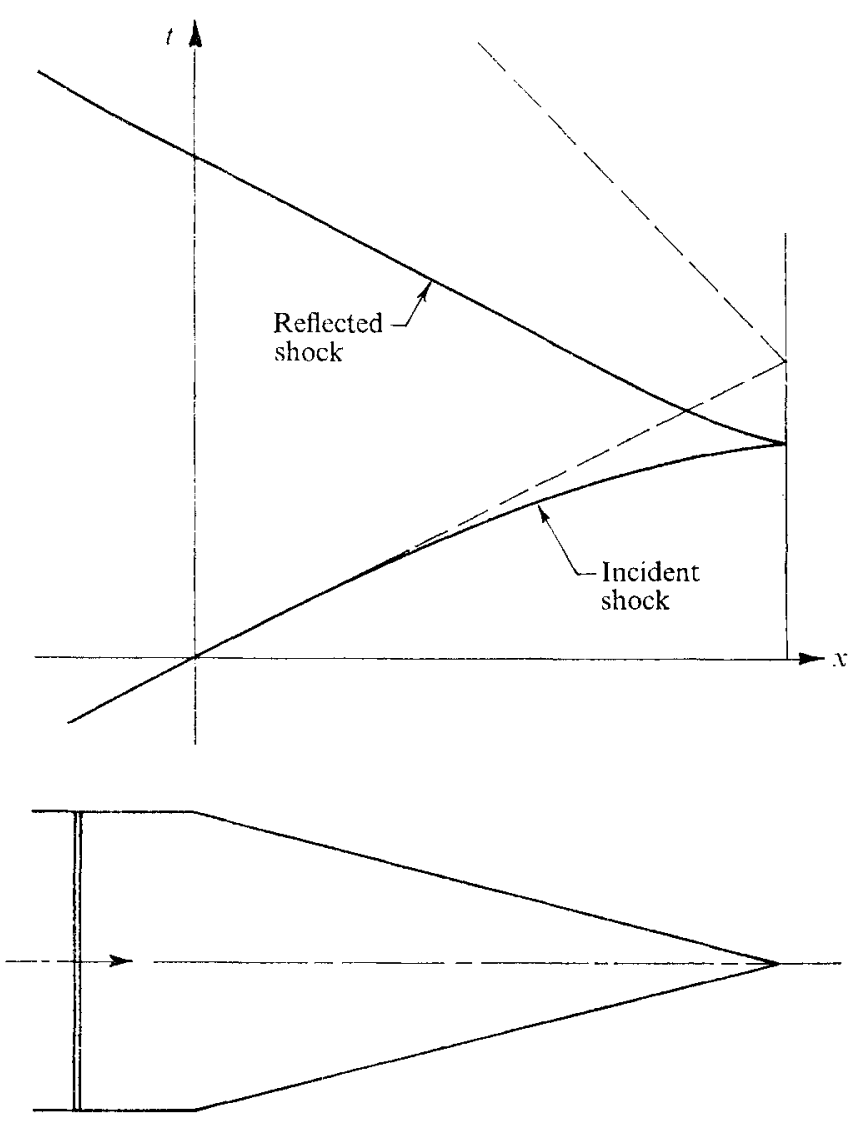

FigURe 1. Initially plane, strong shock wave propagating into a conical convergence. Dashed curve on $x, t$ diagram indicates reflexion of a normal shock wave from a plane end wall.

one-dimensional solution for local shock strength in channels with slowly changing cross-sectional areas. Whitham (1958) obtained an identical solution in an original manner by applying a simplified characteristics method. The Chester-Chisnell-Whitham formulation (hereafter referred to as ' $\mathrm{CCW}$ theory') similarly predicts a power-law increase in the Mach number of a strong shock wave as the surface area of the shock decreases. The power-law exponents found for wedge-shaped and conical channels closely agree with the exponents found in the similarity analysis for converging cylindrical and spherical shocks, respectively. Application of the CCW theory is restricted, however, by the assumption that the interaction between the shock wave and the converging channel is one-dimensional. In an experimental investigation involving a convergence mounted on a conventional shock tube, the possibility of shock diffraction at a discontinuity in wall slope exists. Whitham $(1957,1959)$ developed general equations for shock diffraction problems, and his two-dimensional solution for diffraction by a wedge indicates that Mach reflexion will occur at the entrance of a wedge-shaped or conical channel. The effect of this initial diffraction on the subsequent shock motion within the convergence remained to be determined. 


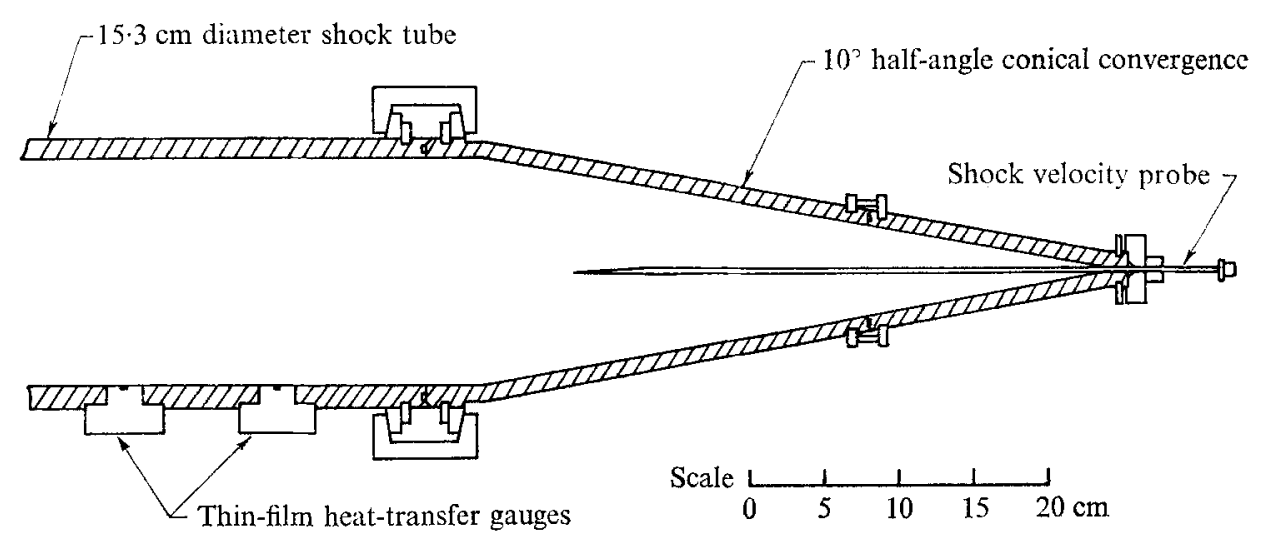

Figure 2. Experimental apparatus.

The few experimental investigations into this shock behaviour have been conducted using various contractions placed on standard shock tubes. Conclusions from these efforts were limited by a general inability to measure accurately shock velocities within the converging channel. Russell (1967) measured shock velocities in a constant-area channel downstream of a conical convergence, then estimated velocities at the exit of the convergence by extrapolation. His estimated values fell below the predictions of the CCW theory, and this disparity increased with increasing Mach number and decreasing pressure. A schlieren system and drum camera were used by both Bird (1959) and McEwan (1968) to obtain shock trajectories within two-dimensional convergences. Local shock velocities were estimated from the slope of the trajectories, and for smooth gradual contractions they found values that roughly compared with the predictions of $\mathrm{CCW}$ theory. $\dagger$ This streak-photograph technique failed to show shock diffraction effects after the initial Mach reflexion at the convergence entrance. Belokin', Petrukhin \& Proskuryakov (1965) noted that the diffraction in a wedge-shaped contraction would be a continuing pattern of Mach reflexions, but their measurements were restricted to examining thermodynamic conditions near the vertex.

This paper summarizes an experimental and theoretical investigation of the behaviour of an initially plane, strong shock wave propagating into a conical convergence. A particular objective of the study was to determine the extent to which the expected diffraction at the entrance affects the subsequent shock motion. Section 2 of the paper is a brief description of the experimental apparatus, which includes a new device for measuring shock velocities within the cone. Section 3 presents complete profiles of the incident shock velocity for two cases of low and high initial Mach number. In $\$ 4$ an approximate solution of Whitham's axisymmetric diffraction equations is obtained for a converging conical geometry. The description of the incident shock motion provided by this solution is then compared with the measured velocity profiles. The investigation was concluded

+ McEwan claimed velocity increases in a parabolic contraction that were greater than CCW theory predictions, and suggested that an optimum geometry might be found. Initial investigations into the effects of wall shaping have been made by Milton \& Archer (1969), Lau (1971) and Skews (1972). 

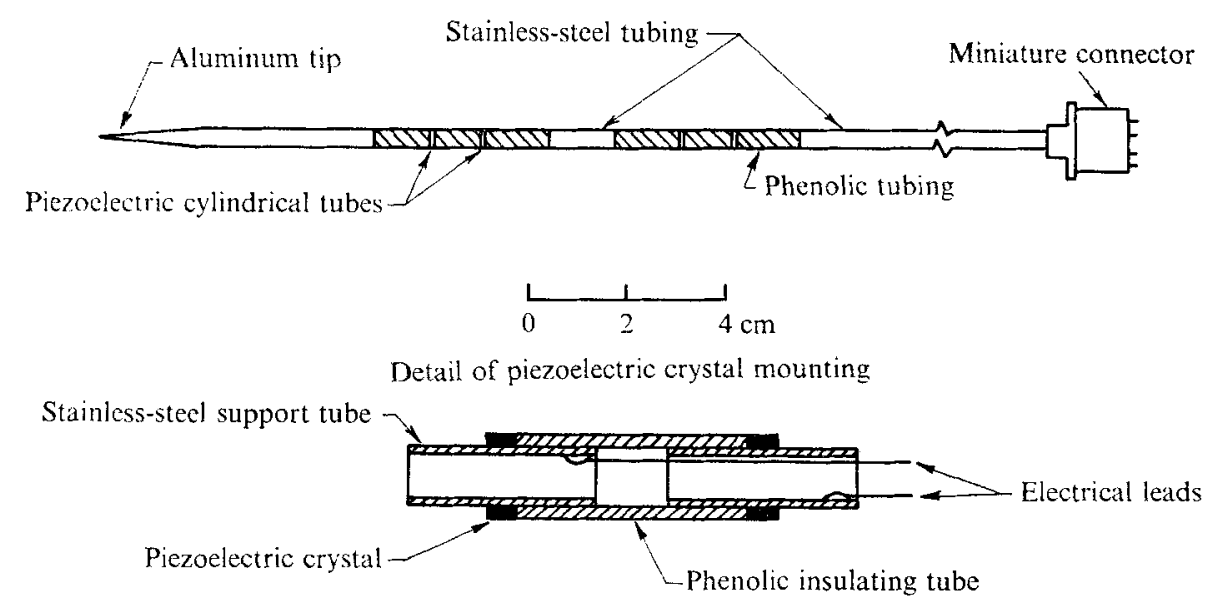

FigURE 3. Shock velocity probe.

by examining the reflected shock behaviour within the cone, and complete profiles of reflected shock velocity are presented in $\S \mathbf{5}$.

\section{Experimental apparatus}

The experiment was performed using a $10^{\circ}$ half-angle conical convergence mounted on the end of a $15.3 \mathrm{~cm}$ diameter, pressure-driven shock tube (figure 2 ). The cone consists of two carefully machined sections, with the final section converging to a minimum diameter of $3.2 \mathrm{~mm}$ (an overall area reduction of $2300: 1)$. A $3 \cdot 2 \mathrm{~mm}$ hole remains at the vertex to allow insertion of instruments. The initial Mach number of the incident shock wave is measured using conventional thin-film heat-transfer gauges mounted upstream of the cone entrance.

A new instrument designed to provide accurate shock velocity measurements along the centre-line of the cone (Setchell 1971) is shown in figure 3 . The probe contains four piezoelectric crystals in the form of cylindrical tubes, each $3.2 \mathrm{~mm}$ in diameter and $1 \mathrm{~mm}$ in length. The crystals are arranged in two closely spaced pairs within the $3.2 \mathrm{~mm}$ diameter, axisymmetric support structure. The probe is inserted into the cone at the vertex and positioned along the axis of symmetry. The piezoelectric crystals are polarized between the inner and outer diameters; the radial compression produced by the shock pressure jump thus generates a sequence of signals as the shock passes the crystal positions along the probe. The signals are fed directly into two dual-beam oscilloscopes and recorded on polaroid oscillograms. Shock velocities are determined by measuring the time interval between signals produced by the adjacent crystals in each crystal pair. The resolution in measuring the time intervals is sufficient to obtain velocities accurate to $3 \%$ or better. The probe was tested in the straight shock tube, where reference velocities could be measured with thin-film side-wall gauges; over a range of shock Mach numbers from 5 to 10 the probe-measured velocities were found to be within $1-4 \%$ of the reference values.

To obtain a complete profile of shock velocity versus distance, a large number 


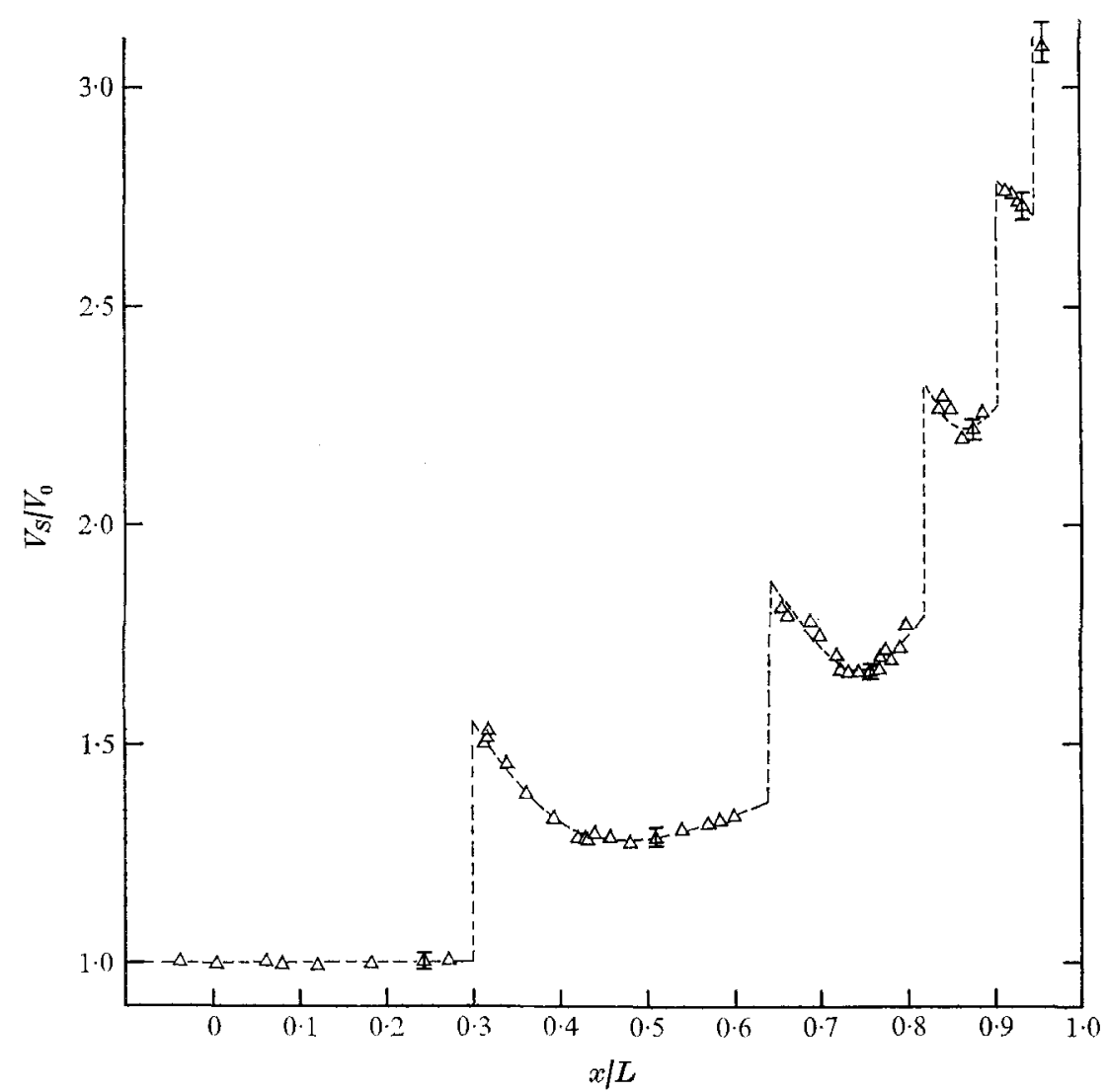

Figure 4. $M_{0}=6 \cdot 0$ incident shock velocity profile. Test gas is argon at $1 \cdot 5$ Torr. Local centre-line shock velocity normalized by the initial shock velocity is plotted versus normalized distance into the convergence $(L$ being defined as the initial cone radius divided by the tangent of the convergence half-angle). Each point is an average of two or more measurements; error bars indicate scatter between measurements.

of runs must be made under identical conditions as the probe position within the

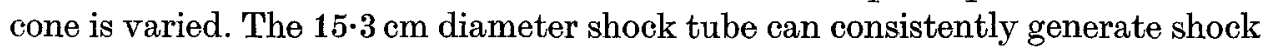
waves whose Mach numbers are reproducible to within 1\% (Smith 1967).

\section{Shock velocity profiles}

\subsection{Mach-six case}

For the first shock velocity profile a relatively low initial Mach number and high test-gas pressure were needed to minimize deviations from ideal-gas behaviour. Measurements made with an initial Mach number $\left(M_{0}\right)$ of $6 \cdot 0$ in argon at a pressure of 1.5 Torr are shown in figure 4 . The data reveal that the shock velocity along the cone centre-line does not display the gradual monotonic increase predicted by the one-dimensional CCW theory for a conical channel (Whitham 1958). Instead there are a number of short intervals in which the centre-line shock exhibits a very rapid acceleration. In the regions between these sudden jumps 

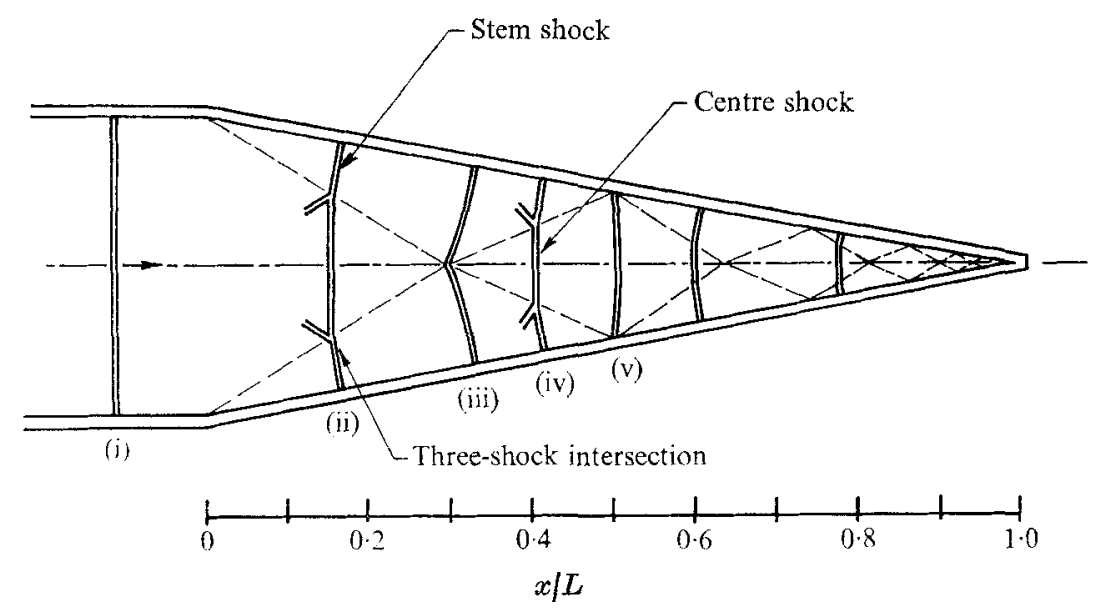

FIGURE 5. Diffraction of the incident shock wave: (i) plane initial shock; (ii) Mach reflexion on the cone wall; (iii) stem-shock intersection on the cone axis; (iv) Mach reflexion on the cone axis; (v) start of the second diffraction cycle. _ _ - , trajectory of the threeshock intersection.

the shock first decelerates then gradually accelerates. The measured velocity for the first $30 \%$ of the convergence is the same as the initial shock velocity. Near the vertex the velocity has increased by a factor of approximately 3 .

The physical interpretation of the measured velocity profile (consistent with the analysis in \$4) is sketched in figure 5. Mach reflexion of the initially plane shock occurs on the convergence wall, $\dagger$ and subsequent diffraction processes continue throughout the length of the cone. The first velocity jump results from the arrival of the three-shock intersection at the cone centre-line (indicated by (iii) in the figure). Beyond this point Mach reflexion of the stem shock on the cone axis occurs, and a 'centre shock' progressively grows until it fills the channel cross-section. The process of Mach reflexion on the cone wall followed by Mach reflexion of the stem shock on the cone axis is then repeated, and this cycle evidently continues until the shock reaches the vertex.

\subsection{Mach-ten case}

In order to observe the possible influence of 'real-gas effects' on the shock diffraction process, a second velocity profile was measured with the initial Mach number increased to $10 \cdot 2$. The test gas was again argon at a pressure of $1 \cdot 5$ Torr. For these initial conditions, the shock should be sufficiently strengthened after the first diffraction cycle to produce significant ionization in the heated argon. The data are plotted in figure 6 and show the same general features as are found in the Mach-six case until the third stem-shock intersection on the cone axis, where local Mach number jumps from $19 \cdot 1$ to $23 \cdot 2$. Beyond this point the high

$\dagger$ In Mach reflexion the shock strength and propagation direction are discontinuous at the intersection of the original shock, the stem shock, and the weak reflected shock. The three-shock intersection moves outwards from the channel wall as the shock motion progresses. 


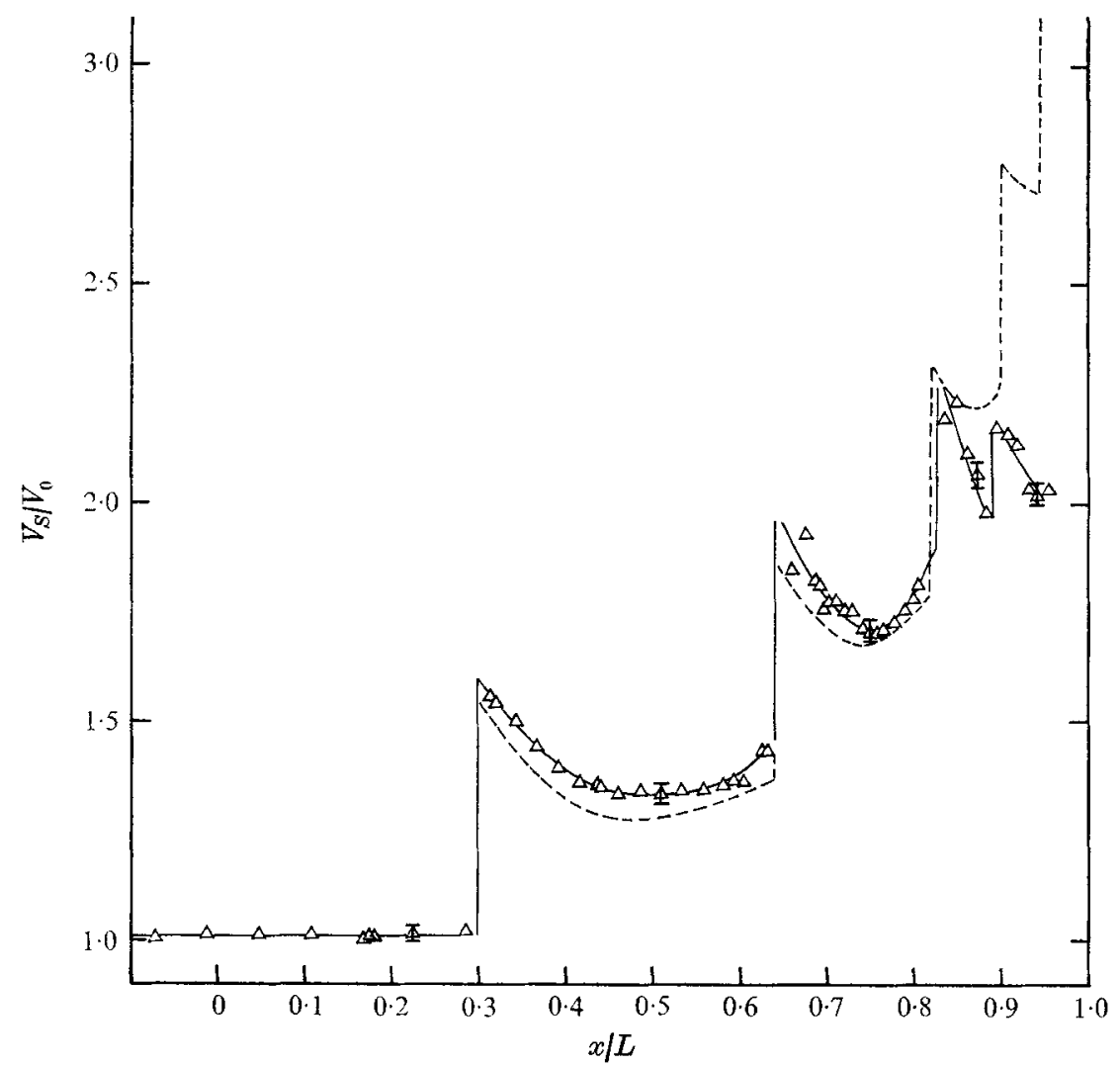

Frgure 6. $M_{0}=10.2$ incident shook velocity profile. Test gas is argon at 1.5 Torr. Each point is an average of several measurements, and error bars indicate scatter. - - $M_{0}=6.0$ data.

Mach number data show a rapid decline in the shock velocity. The jump at the fourth stem-shock intersection is very weak, and a fifth intersection is not observed.

\subsection{Variation of initial Mach number and pressure}

The shock diffraction theory (discussed in \$4) predicts that the local shock velocity at any particular location within the cone should scale with the initial velocity. Because the theory assumes ideal-gas behaviour, a breakdown in this scaling is expected at higher Mach numbers and lower pressures, when real-gas effects become important. Figure 7 shows a series of measurements made at fixed locations within the cone while the initial Mach number was varied from $6 \cdot 0$ to $10 \cdot 2$. The initial pressure was either 1.5 Torr or 0.5 Torr. As was expected from the complete velocity profile measurements, no significant scaling variations are observed for a pressure of 1.5 Torr except at a position between the third and fourth stem-shock intersections $(x / L=0 \cdot 86)$. At this location the scaling begins to fail at a local shock Mach number between 18.7 and 19.8. For a pressure of 0.5 Torr the measured velocities at the same location are unexpectedly lower and show no variation with initial Mach number. The highest local Mach number measured at this pressure is $21 \cdot 1$. 


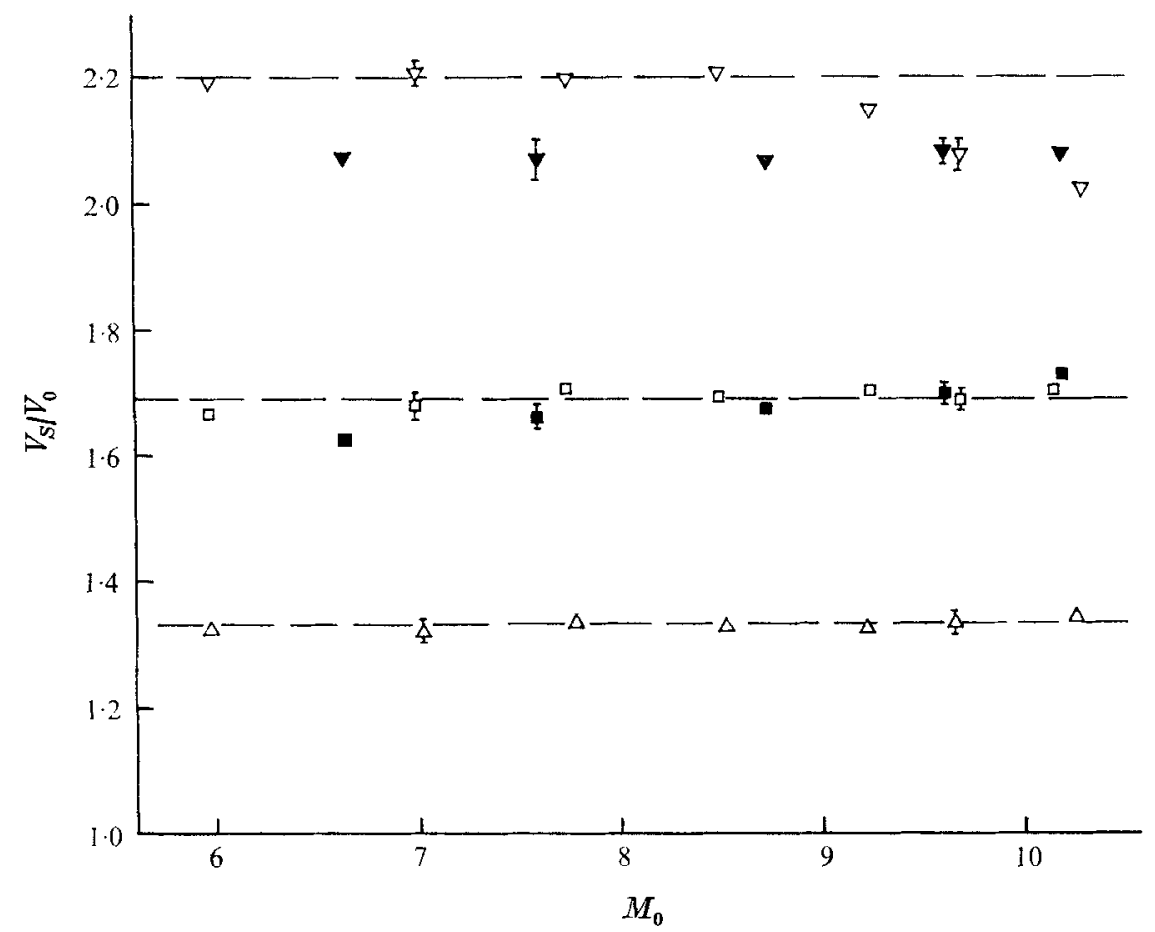

Frgure 7. Variation of initial Mach number and pressure. Initial pressure: open symbols, 1.5 Torr (argon); solid symbols, 0.5 Torr. Measurement position $(x / L): \triangle, 0.580 ; \square$, $\square, 0.743 ; \nabla, \nabla, 0.862$.

\section{Axisymmetric shock diffraction solution}

\subsection{Whitham's diffraction theory}

Whitham $(1957,1959)$ formulated an approximate theory for shock dynamics problems in which disturbances to the flow are treated as a wave propagation on the shock. In two-dimensional problems the successive shock positions and rays locally normal to these positions are used as orthogonal co-ordinates. One relation between the local shock Mach number and the distance between adjacent rays follows from the geometry and a second is obtained from the CCW theory (Whitham 1958) by assuming that adjacent rays act like solid channel walls. Combining the two relations results in a second-order hyperbolic differential equation which predicts that disturbances on the shock display nonlinear wave motion analogous to waves in one-dimensional unsteady gasdynamics. Mach reflexion at an inward wall inclination initially appears as a compressive 'wave" on the incident shock, but this wave quickly breaks owing to the nonlinearity to form a 'shock-shock'. This shock-shock represents the three-shock intersection characteristic of Mach reflexion, and the theory is correct to the extent that the effects of the third (reflected) shock are negligible.

In axisymmetric problems the formulation is basically the same, except that conventional cylindrical co-ordinates $(x, r)$ prove to be more convenient than independent variables based on shock positions and rays. The diffraction 


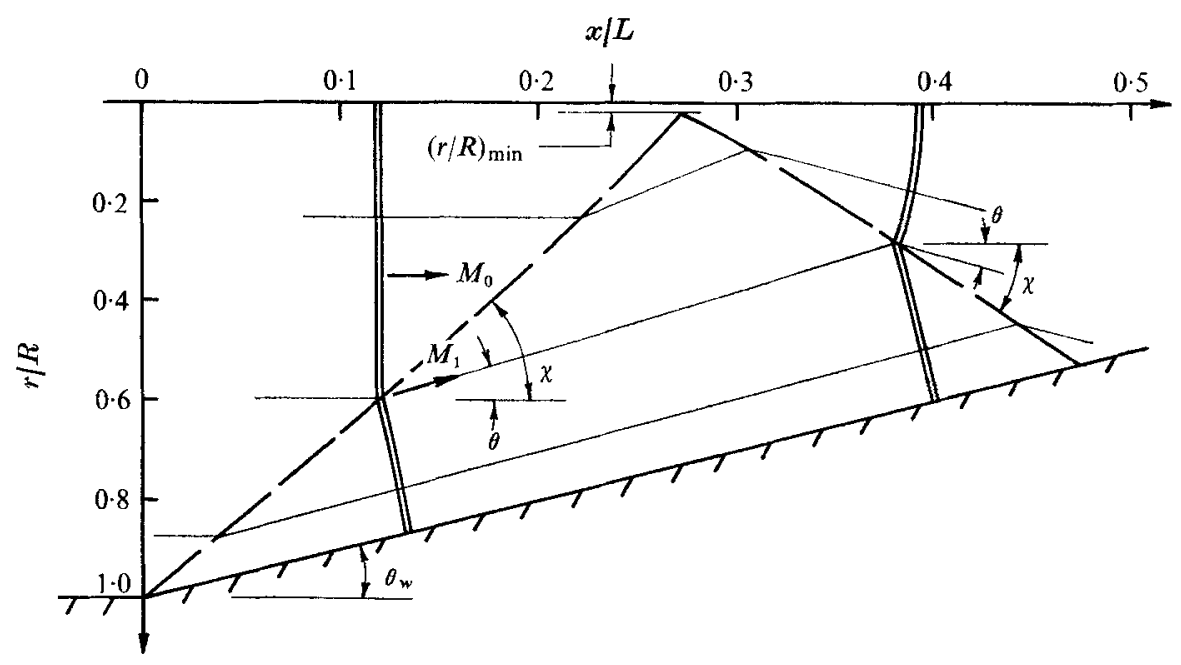

Figure 8. Axisymmetric diffraction geometry. Dashed lines are shock-shock trajectories; thin solid lines indicate rays. $L=R / \tan \theta_{w}$.

geometry and the variables used in the analysis that follows are illustrated in figure 8 . The shock surface at a particular time $t$ is described by $a_{0} t=\alpha(x, r)$, where $a_{0}$ is the sound speed in the undisturbed gas. If $M$ is the local shock Mach number and $\theta(x, r)$ is the local angle between a ray and the symmetry axis, then $\alpha_{x}=\cos \theta / M$ and $\alpha_{r}=\sin \theta / M$, so that

$$
\frac{\partial}{\partial x}\left(\frac{\sin \theta}{M}\right)-\frac{\partial}{\partial r}\left(\frac{\cos \theta}{M}\right)=0
$$

If $A$ is proportional to the area of a ray tube, then for purely geometrical reasons $\dagger$ $A$ must satisfy

$$
\frac{\partial}{\partial x}\left(\frac{r \cos \theta}{A}\right)+\frac{\partial}{\partial r}\left(\frac{\mathrm{r} \sin \theta}{A}\right)=0
$$

Jump conditions across the surface of successive shock-shock positions (hereafter called the shock-shock trajectory) are obtained by considering a narrow ray tube which intersects this surface. The continuity of $\alpha$ and the conservation of $M \nabla \alpha / A$ result in the following:

$$
\begin{aligned}
& \tan \left(\theta_{1}-\theta_{0}\right)=-\frac{\left(\left(M_{1} / M_{0}\right)^{2}-1\right)^{\frac{1}{2}}\left(1-\left(A_{1} / A_{0}\right)^{2}\right)^{\frac{1}{2}}}{1+\left(A_{1} M_{1} / A_{0} M_{0}\right)} \\
& \tan \left(\chi-\theta_{0}\right)=\left(\frac{\left(M_{1} / M_{0}\right)^{2}-1}{1-\left(A_{1} / A_{0}\right)^{2}}\right)^{\frac{1}{2}}
\end{aligned}
$$

where the subscripts 0 and 1 refer to conditions ahead of and behind the shockshock trajectory, respectively, and $\chi(x, r)$ is the local angle between the trajectory and the axis of symmetry. A final relation between $A$ and $M$ follows from the CCW theory; in the strong shock limit

$$
A / A_{0}=\left(M_{0} / M\right)^{n}, \quad n=\frac{\gamma+2}{\gamma}+\left(\frac{2 \gamma}{\gamma-1}\right)^{\frac{1}{2}},
$$

$\dagger$ Detailed derivations of (2)-(5) are given in the cited papers of Whitham. 
where $\gamma$ is the ratio of specific heats. After (5) has been used to eliminate $A$, (1) and (2) form a set of equations for the two dependent variables, $M$ and $\theta$, subject to the boundary condition at the wall $\left(\theta=\theta_{w}\right)$ and the jump conditions given by (3) and (4). To solve this system of equations, numerical techniques employing the method of characteristics are necessary (analogous to the procedures used in axisymmetric steady supersonic flow problems).

\subsection{Solution for the initial shock-shock trajectory}

The formidable computational work required to solve the full equations can be avoided by applying the simplified characteristics method used by Whitham (1958) in his formulation of the CCW theory. + This application is motivated by the analogy between Whitham's shock-shocks in two-dimensional problems and ordinary one-dimensional shock wave propagation. For example, the linear shockshock trajectory resulting from diffraction by a wedge (as viewed in a Cartesian plane) corresponds to the linear path of a shock wave produced by a piston impulsively started into steady motion (as viewed in a distance-time plane). When extended to the present axisymmetric case, this analogy calls for the initial shock-shock trajectory to behave like a shock wave produced by a converging cylindrical piston.

The first step in the solution is to write (1) and (2) in characteristic form (after eliminating $A$ ):

$$
d \theta \pm \frac{n^{\frac{1}{2}}}{M} d M+\frac{\tan \theta}{1 \pm n^{\frac{1}{2}} \tan \theta} \frac{d r}{r}=0 \quad \text { on } \quad \frac{d r}{d x}=\frac{1 \pm n^{\frac{1}{2}} \tan \theta}{ \pm n^{\frac{1}{2}}-\tan \theta}
$$

Equation $(6 b)$ is a differential relation that holds on characteristics which originate at the cone wall and arrive at the shock-shock trajectory. Following Whitham's method, this equation is applied to local shock wave conditions immediately behind the shock-shock. These conditions are then related to the initial values ahead of the shock-shock by means of the jump relations (3) and (4). After substitution and rewriting, the equation for the trajectory of the first shock-shock reduces to

$$
\begin{gathered}
\frac{r}{R}=\exp -\left[\int_{Z_{2-D}}^{Z} g(u)\left(G(u)+\frac{n^{\frac{1}{2}}}{u}\right) d u\right] \text { with } g(u)=n^{\frac{1}{2}}+\frac{u\left(u^{n-1}+1\right)}{\left(u^{2}-1\right)^{\frac{1}{2}}\left(u^{2 n}-1\right)^{\frac{1}{2}}}, \\
G(u)=\frac{u^{n-1}+1}{\left(u^{n+1}+1\right)^{2}} \frac{\left(u^{2}-1\right)^{\frac{1}{2}}}{\left(u^{2 n}-1\right)^{\frac{1}{2}}}\left[n+u^{2} \frac{u^{2 n-1}}{u^{2}-1}+(n-1) \frac{u^{2 n}-1}{u^{n-1}+1}\right] \\
Z=M_{1}(x, r) / M_{0}, \quad Z_{2-D}=\cos \left(\chi_{2-D}-\theta_{w}\right) / \cos \chi_{2-D}
\end{gathered}
$$

where $R$ is the initial cone radius, $u$ is a dummy variable for Mach number and $\chi_{2-D}$ is given by Whitham's solution for shock diffraction by a wedge with halfangle $\theta_{w}$. The computational procedure is as follows.

(i) A series of values for $M_{1} / M_{0}$ are chosen and equation (7) is integrated numerically to find the corresponding $r / R$.

(ii) For each pair of values for $M_{1} / M_{0}$ and $r / R, \chi$ follows from equations (4) and (5).

$\dagger$ The authors are indebted to Professor Whitham for suggesting this simplification. 


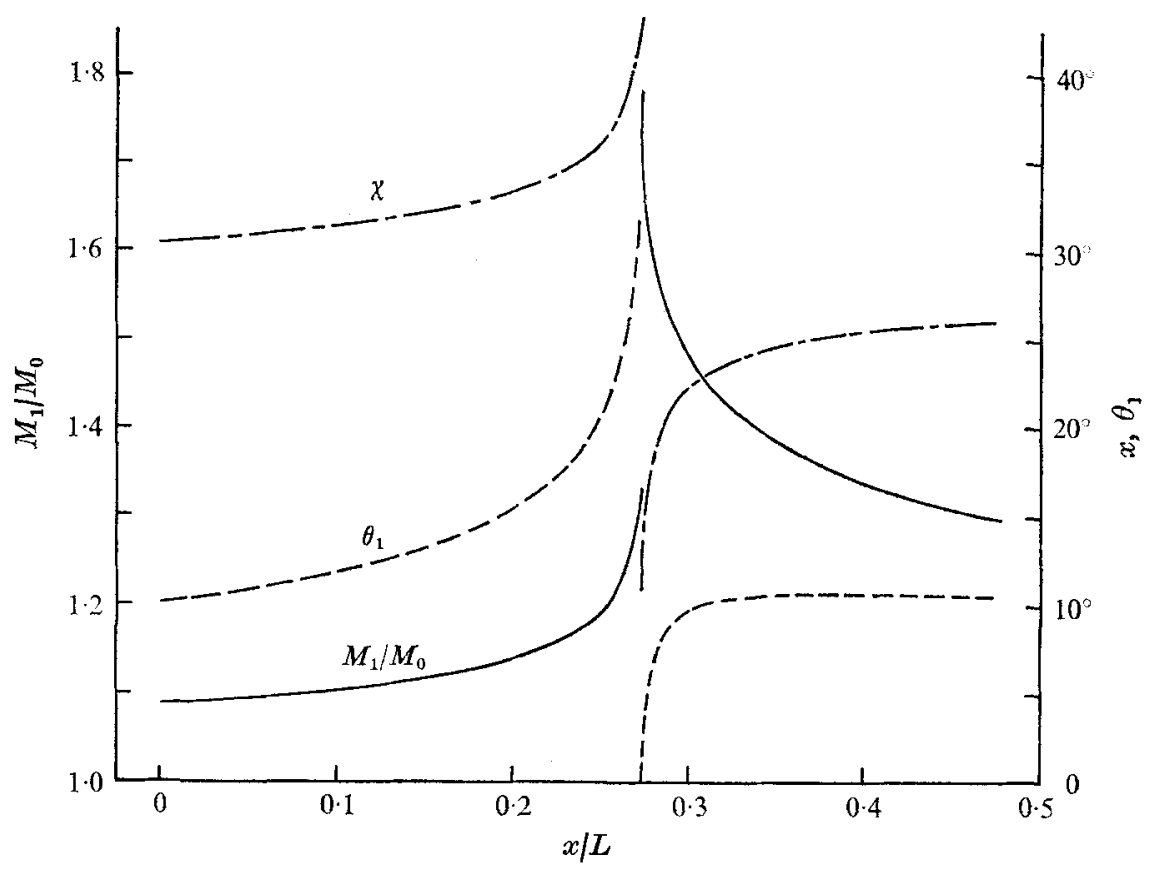

FIGURE 9. Asixymmetric diffraction solution for conditions along the first two shock-shock trajectories. The ratio of specific heats is $\frac{5}{3}, \theta_{w}$ is $10.17^{\circ}$ and $(r / R)_{\min }$ is 0.021 (shock velocity probe radius). from

(iii) For each set of $M_{1} / M_{0}, r / R$ and $\chi$ values, the corresponding $x / R$ is obtained

$$
\frac{x}{R}=\int_{r / R}^{1} \frac{1}{\tan \chi} d(r / R) .
$$

These calculations give the initial shock-shock trajectory and the stem-shock Mach number along this curve. The ray inclination along the trajectory $\left(\theta_{1}\right)$ is found at each point using (3). Information on conditions at other points along the stem shock or in the flow behind the shock is not provided by the solution.

The computed shock-shock trajectory is shown in figure 8 and local shock wave conditions along the trajectory are plotted in figure 9 . The curves show that $M_{1}, \theta_{1}$ and $\chi$ all increase monotonically as the cone axis is approached. In the limit $r / R \rightarrow 0, M_{1} \rightarrow \infty$ while $\theta_{1}$ and $\chi$ approach $\frac{1}{2} \pi$. This singular behaviour is evident in the numerical calculations only for extremely small values of $r / R . \dagger$

\subsection{Extension beyond the stem-shock intersection on the cone axis}

An analytical procedure for bridging the singularity on the cone axis has not been obtained. Instead, the solution is extended by assuming that a solid boundary exists at some small value of $r / R$ (figure 8 ). The minimum $r / R$ chosen for the calculations shown in figure 9 corresponds to the radius of the shock velocity

$\dagger$ For example, when the shock-shock trajectory reaches $r / R=10^{-4}$ the solution gives $\theta_{1}=63 \cdot 4^{\circ}, \chi=64 \cdot 6^{\circ}$ and $M_{1} / M_{0}=2 \cdot 33$. 
probe used in the experiment. The initial values of $M_{1}$ and $\chi$ required for computation of the second shock-shock trajectory are specified by assuming local two-dimensional diffraction at the minimum value of $r / R$. After the appropriate change in $Z_{2-D}$, the necessary calculation is again numerical integration of (7). For simplicity the origin of the co-ordinate system is shifted to the start of the second trajectory. A basic difficulty in the procedure now arises from the fact that the solution for the initial shock-shock trajectory does not provide information about local shock wave conditions at other points along the stem shock. Consequently, the initial conditions ahead of the second shock-shock $\left(M_{0}, \theta_{0}\right.$ and $\left.A_{0}\right)$ are unknown. This difficulty is treated by assuming that the actual variation in the shape and strength of the stem shock can be approximated by taking the rays to be straight and the Mach number between successive rays to be constant $\dagger$ in the region between the first and second shock-shock trajectories. With this assumption the computation is similar to that for the first shock-shock trajectory, and results for the $10^{\circ}$ half-angle cone are plotted in figure 9 . The curves show that near the cone axis the shock-shock conditions change rapidly, with $\theta_{1}$ and $\chi$ increasing while $M_{1}$ falls. These trends continue more gradually as the shock-shock approaches the cone wall.

The numerical calculation is discontinued when the second shock-shock reaches the wall, although remaining shock-shock trajectories can be computed in a similar manner. Subsequent cycles of Mach reflexion on the cone wall followed by Mach reflexion on the axis are approximated by assuming that in each cycle the centre shock is initially plane and uniform. The diffraction is then geometrically similar to the first cycle, and the initial calculations are applied after appropriate scale changes. This procedure is made possible by the fact that the solution does not depend on the value of the initial Mach number $M_{0}$, provided that this value is large enough $\left(M_{0} \gtrsim 3\right)$ for $(5)$ to be valid.

An interesting final note on the diffraction solution would be a comparison between the Mach number averaged over the entire shock front (centre shock and stem shock) at each position within the cone and the Mach number variation predicted by the one-dimensional CCW theory. Such a comparison can be made easily for a wedge-shaped convergence, $\ddagger$ and in that case the averaged diffraction solution reduces identically to the curve predicted by CCW theory. Unfortunately, the simplified characteristies method used in the present axisymmetric case does not provide sufficient information to calculate the average Mach number. Rough estimates of such an average are found to depend on the choice and scaling of the minimum value of $r / R$. If $R^{\prime}$ represents the initial cone radius at the start of

\footnotetext{
† It might appear more consistent to assume the Mach number between successive rays satisfies (5). This would result in a gradually increasing Mach number along the rays, since the divergence of the straight rays and their decreasing radial position have the combined effect of slowly reducing $A$. The actual variation of $A$ due to ray curvature is not known, however, and the approximation as stated gives better agreement with experimental results.

‡ The solution for a wedge-shaped convergence follows directly from the solution for diffraction by a wedge (Whitham 1957), since the plane of symmetry acts like a solid boundary. A cyclic pattern of Mach reflexions occurs, but in this case all shock-shock trajectories, stem shocks and centre shocks are straight.
} 


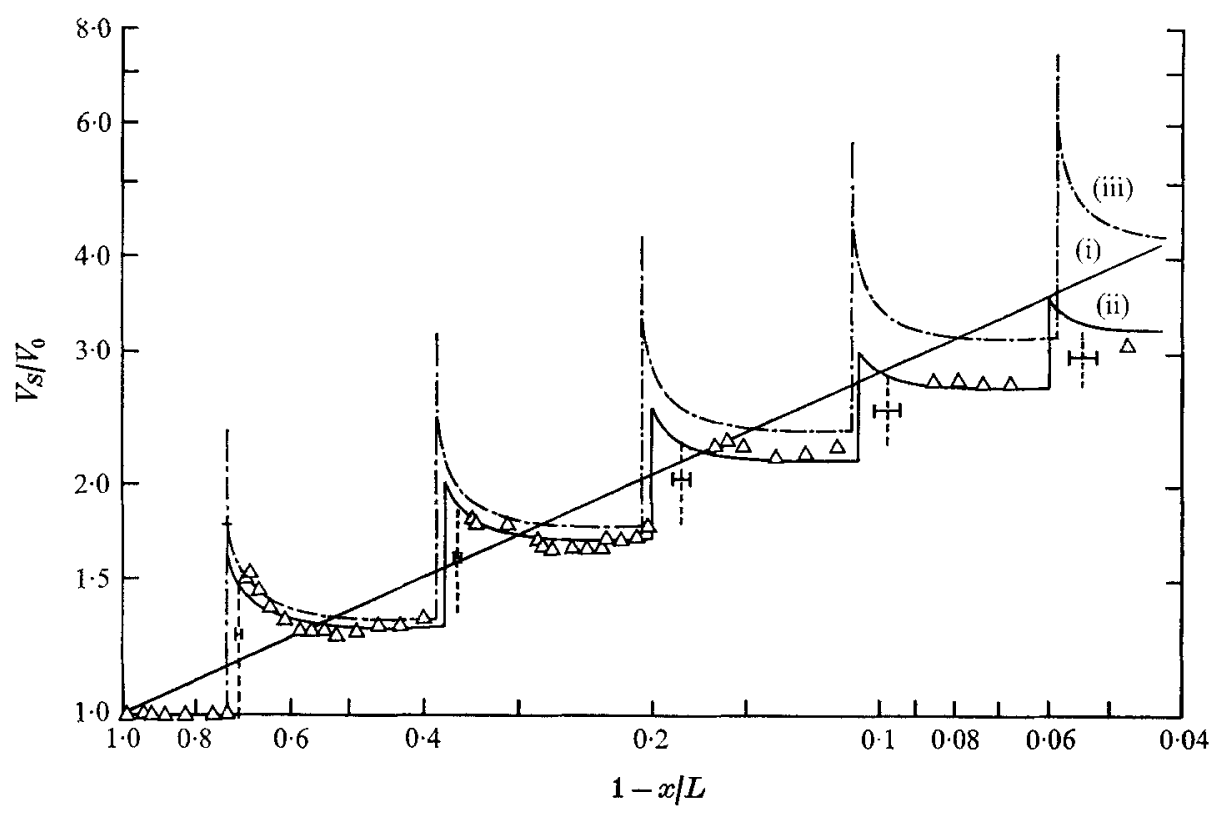

Figure 10. Comparison between (i) the one-dimensional CCW theory; (ii) the diffraction solution with $r_{\min }$ equal to the velocity probe radius; (iii) the diffraction solution with $\left(r / R^{\prime}\right)_{\min }$ equal to 0.003 ; (iv) the $M_{0}=6.0$ measurements. Vertical dashed lines indicate measured velocity jumps at the stem-shock intersections on the axis; corresponding error bars indicate experimental uncertainty in position.

each diffraction cycle, then setting $\left(r / R^{\prime}\right)_{\min }$ equal to a small fixed constant results in better agreement with the CCW theory than if $r_{\mathrm{min}}$ is fixed at some value such as the radius of the velocity probe. This difference is illustrated in figure 10 , and will be discussed further in the following section.

\subsection{Comparison between the diffraction solution and the shock velocity measurements}

During each Mach reflexion process, the variation in Mach number over the stem-shock surface should be small compared with the abrupt change at the three-shock intersection. $\dagger$ The centre shock Mach number along the second shock-shock trajectory is therefore a good approximation to the corresponding Mach number on the cone axis (figure 8 ), and the diffraction solution can be compared with the velocity probe measurements. As is shown in figure 10, the basic characteristics observed in the experiment are predicted by the diffraction solution with $r_{\min }$ set equal to the probe radius. In the first diffraction cycle the stem-shock intersection on the cone axis is predicted to occur slightly ahead of the position observed experimentally, and the resulting shock velocity jump is somewhat larger. The solution qualitatively predicts the observed deceleration following the stem-shock intersection, but not the gradual acceleration prior to the next intersection. The close resemblance between this and subsequent diffraction cycles supports the assumption of geometrical similarity for the diffraction process after the first cycle. A comparison between the solution and the

$\dagger$ This is evident in the solution for shock diffraction by a solid cone (Whitham 1959). 
$M_{0}=10.2$ measurements shows greater disparities (as indicated by the velocity profiles in figure 6), but this is expected since the theoretical model assumes perfect-gas behaviour.

The low Mach number measurements show that the velocity increase at the stem-shock intersections (the difference between the initial and final values) is nearly constant. This constancy is also predicted by the diffraction solution with $r_{\min }$ set equal to a fixed constant (such as the probe radius). However, the solution with $\left(r / R^{\prime}\right)_{\min }$ fixed at some small value (curve (iii) in figure 10 ) predicts that the ratio of final to initial velocities at the intersections is constant, resulting in much higher velocities near the vertex. Since estimates of the average Mach number using this method of scaling $r_{\min }$ compare more favourably with CCW theory, this suggests that greater shock strengthening than that indicated by the present measurements might occur in the convergence if the velocity probe were not present. $\uparrow$

\section{Reflected shock behaviour}

\subsection{Shock velocity profiles}

The behaviour of the reflected shock within the convergence is governed by both the divergent geometry and the complex flow generated by the incident shock. The concluding phase of the present investigation was an examination of this complicated shock motion. The velocity probe was found to respond adequately to the reflected shock, thereby enabling shock velocity measurements to be made in the same manner as for the incident shock. An additional probe with an extended support rod was used to obtain data as far upstream as $13 \mathrm{~cm}$ into the constant-area shock tube. Complete velocity profiles for the two cases of Machsix and Mach-ten initial shock waves are shown in figure 11. The most outstanding feature of the profiles is the nearly constant velocity observed over most of the convergence length. Small variations in the velocity are apparent near the vertex in both cases, and near the cone entrance in the $M_{0}=6.0$ case. Upon reaching the constant-area channel the shock decelerates towards the velocity corresponding to reflexion from a plane end wall. $\ddagger$ In the $M_{0}=6 \cdot 0$ case the reflected shock could not be detected for $x / L>0 \cdot 84$, but a second reflected shock was observed in the region $0.74<x / L<0.92$ propagating with nearly the same velocity as the primary shock. The relative position of this second shock is indicated in figure 12, which shows the distance-versus-time behaviour of both the incident and reflected shock waves.

\subsection{Comparison with the Guderley similarity solution}

The Guderley similarity solution for an imploding spherical shock wave (Guderley 1942; Butler 1954) predicts that the velocity of the subsequent reflected shock will vary with radial distance according to a simple power law. As is indicated

$\dagger$ Nevertheless, in the experiment the diffraction process was found to be insensitive to the presence of the probe at the first stem-shock intersection. The probe dimensions prevented a similar investigation at later intersections.

$\ddagger$ The real-gas calculations of Arave \& Huseby (1962) were used to find the velocities for reflexion from a plane end wall. 


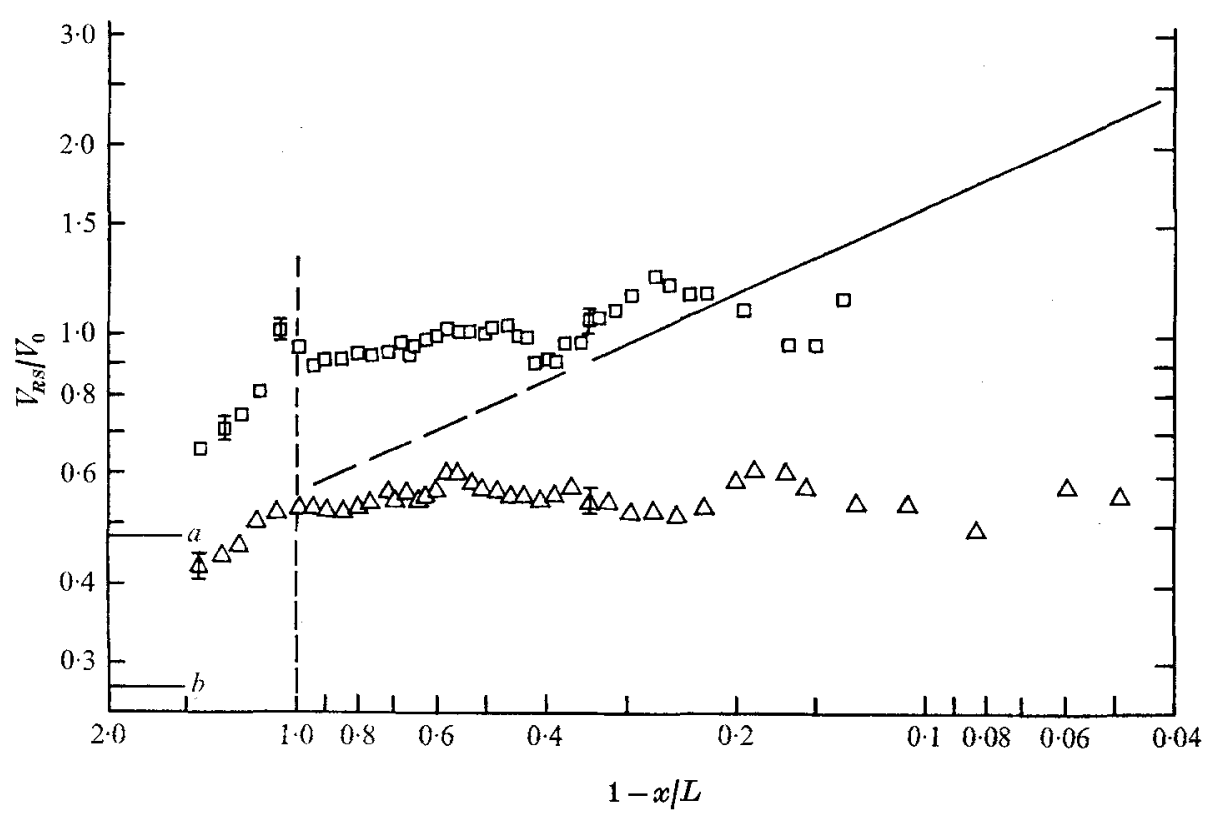

FIGURE 11. Reflected shock velocity measurements: $\square, M_{0}=6.0 ; \Delta, M_{0}=10 \cdot 2$. Each point is an average of two or more measurements; error bars indicate scatter between measurements. The linear curve is the Guderley similarity solution with constants chosen to match the $M_{0}=6.0$ incident shock trajectory. $a$ and $b$ indicate velocities corresponding to reflexion from a plane end wall for $M_{0}=6 \cdot 0$ and $10 \cdot 2$ respectively.

in figure 11, this power law obviously does not compare with the nearly constant shock velocity found in the experiment. The dissimilarity between the measurements and the similarity solution is primarily a consequence of the finite length of the cone, which results in fundamental differences between the experimental flow conditions and the conditions assumed in the similarity analysis. This can be shown by using the similarity solution for the flow behind the incident shock to calculate approximate particle paths for fluid initially within the convergence. Particle paths originating at the cone entrance are drawn in figure 12, and indicate that for $x / L \lesssim 0.66$ in the $M_{0}=6.0$ case, and for $x / L \lesssim 0.75$ in the $M_{0}=10 \cdot 2$ case, the reflected shock propagates into fluid originally set into uniform motion by the incident shock wave in the constant-area channel upstream of the cone. In the similarity analysis the fluid in these regions has supposedly undergone a gradual continuous compression after initially experiencing a much weaker shock, resulting in conditions ahead of the reflected shock that progressively deviate from the experimental flow conditions. A simplified model for the actual flow ahead of the reflected shock, together with an approximate solution for the corresponding shock motion in the $M_{0}=6 \cdot 0$ case, can be found in the appendix.

\section{Conclusions}

A comprehensive investigation has been made of the shock dynamics within a $10^{\circ}$ half-angle conical convergence mounted on a conventional shock tube. 


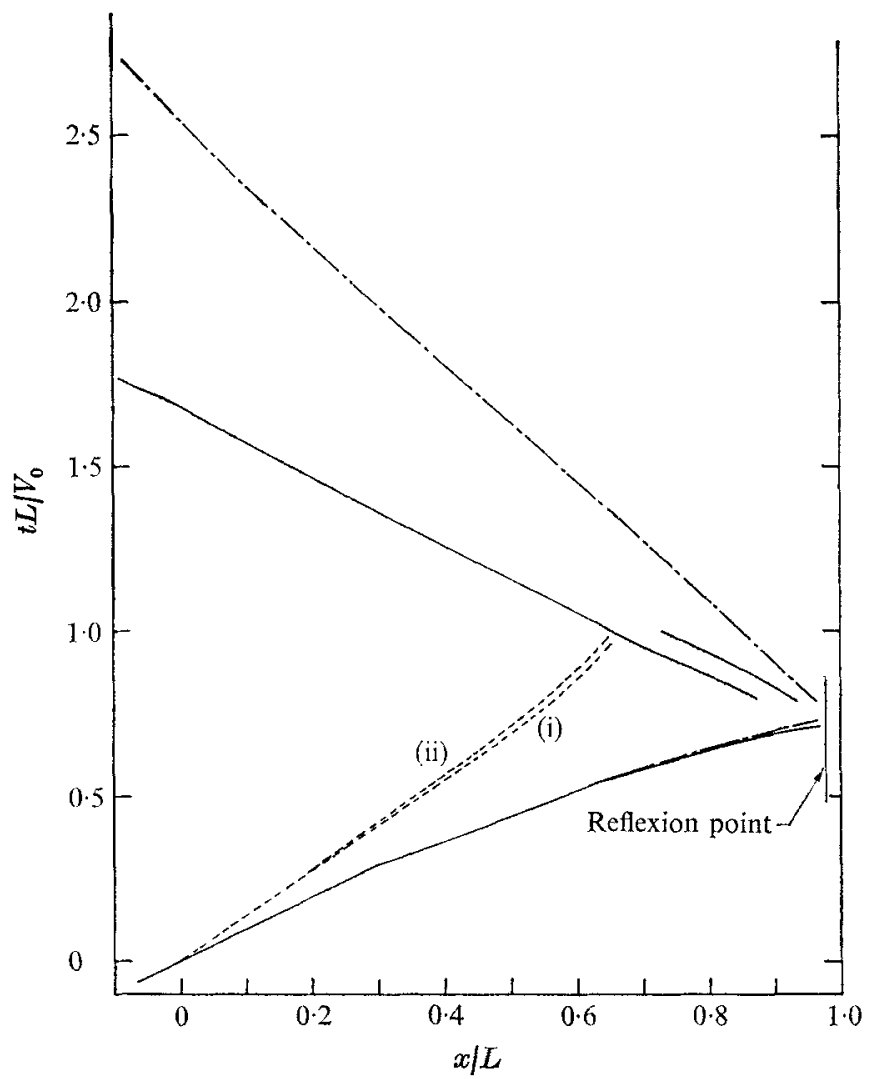

Figure 12. Incident and reflected shock trajectories: -,$M_{0}=6.0$ data; --- , $M_{0}=10 \cdot 2$ data. (i) and (ii) are approximate particle paths for the low and high Mach number cases, respectively, that were computed using the similarity solution.

Measured shock velocity profiles show that the incident shock behaviour is dominated by cyclic diffraction processes which originate at the entrance of the cone. During each diffraction cycle the shock first undergoes Mach reflexion on the cone wall, then Mach reflexion on the axis. These cycles evidently persist until the shock reaches the vertex, where the measured velocity has increased by as much as a factor of three. Real-gas effects, enhanced by increasing the initial Mach number and decreasing the pressure, apparently alter the shock wave behaviour only in the region near the vertex.

The basic features of the incident shock behaviour are described analytically by applying Whitham's axisymmetric shock diffraction equations (Whitham 1959) to the converging conical geometry. An approximate solution is obtained by using the simplified characteristics method applied by Whitham in his formulation of the CCW theory (Whitham 1958). The first two shock-shock trajectories are computed numerically, and subsequent diffraction cycles are assumed to be geometrically similar to the initial cycle.

Measured velocity profiles for the reflected shock within the cone show that the shock velocity is nearly constant throughout most of the convergence length. This behaviour obviously contrasts with the power-law decline in velocity 


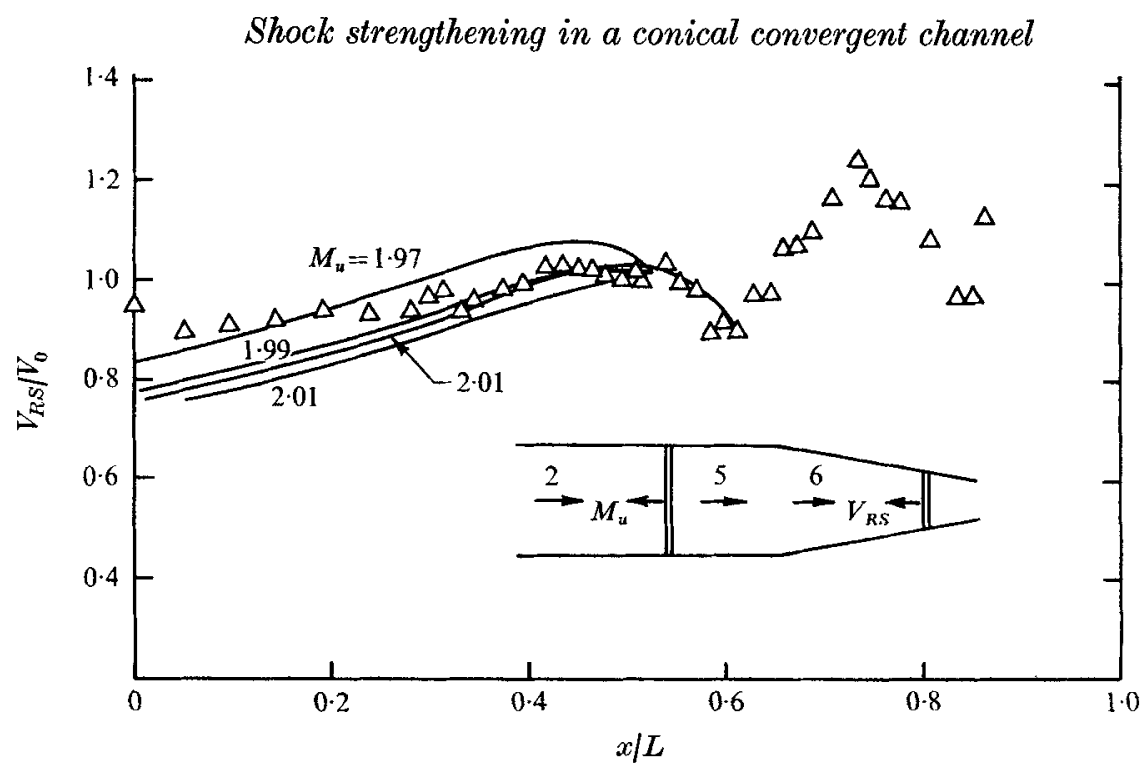

FIgure 13. Reflected shock propagating into steady subsonic flow. Region 2, supersonic flow generated by the initial shock; region 5, steady uniform subsonic flow; region 6 , steady isentropic compression. Data points are $M_{\mathfrak{0}}=6.0$ measurements.

predicted by the Guderley similarity solution (Guderley 1942; Butler 1954). Simple arguments show that this disparity is primarily a consequence of the finite length of the cone, which results in fundamentally different experimental flow conditions ahead of the reflected shock.

\section{Appendix. Reflected shock propagating into steady subsonic flow}

An approximate analytical description of the reflected shock motion can be obtained by assuming a simplified model for the flow of fluid initially outside the cone entrance. The Mach numbers of the flow behind the Mach-six and Machten initial shock waves are $1 \cdot 26$ and $1 \cdot 31$, respectively. At these Mach numbers the supersonic flow near the shock tube wall cannot be turned through the $10^{\circ}$ angle at the cone entrance by means of a stationary oblique shock. Consequently, as the initial shock enters the cone an upstream-facing shock locally normal to the wall must form outside the entrance. $\dagger$ An unsteady sonic surface behind this shock separates subsonic flow adjacent to the wall from the central supersonic stream. The subsonic region grows as the upstream-facing shock propagates outwards from the cone entrance; eventually this region fills the channel crosssection if the sonic surface reaches the axis. A simple one-dimensional flow model which assumes that a uniform subsonic region is created by a plane upstreamfacing shock is pictured in figure 13. The upstream shock is assumed to propagate at constant velocity, resulting in a subsonic flow which subsequently undergoes a steady isentropic compression in the convergence. The reflected shock is assumed to start at some position upstream of the sonic point for this flow with an initial velocity given by the velocity probe measurements.

$\dagger$ This shock is an extension of the third (reflected) shock formed in the initial Mach reflexion on the cone wall. 
The reflected shock motion is found using Whitham's simplified characteristics method for one-dimensional shock propagation into non-uniform media (Whitham 1958) in a manner suggested by Chester (1960). The differential relation for flow variables along characteristics overtaking the reflected shock trajectory is applied to flow conditions immediately behind the shock. The Rankine-Hugoniot relations are then used to couple these conditions to the known distribution of flow variables in the steady compression ahead of the shock. The result is a first-order ordinary nonlinear differential equation for shock Mach number (or velocity) as a function of position that can be numerically integrated. The curves shown in figure 13 were obtained by performing the integration for several possible steady flow situations ahead of the $M_{0}=6 \cdot 0$ reflected shock. The upstream-facing shock was required to be fairly strong (Mach number $\sim 2$ ) in order for the predicted sonic point of the subsonic compression to be sufficiently near the cone vertex. Although the theoretical curves show a gradual deceleration of the reflected shock within the convergence, the comparison with experimental results is still far better than can be made with the similarity solution. $\uparrow$ It should be noted that Whitham's method neglects modification of the shock motion by re-reflected disturbances, and in the assumed flow model such disturbances could be important.

\section{REFERENCES}

Arave, R. \& Huseby, O. 1962 Boeing Rep. D2-11238.

Belokin', V. A., Petrukhin, A. I. \& Proskuryakov, V. A. 1965 Soviet Phys. J.e.t.P. (Eng. Trans.) 21, 33.

Bird, G. A. 1959 J. Fluid Mech. 5, 60.

Buther, D. S. 1954 Armament Res. Establishment Rep. 54/54.

Chester, W. 1954 Phil. Mag. 45 (7), 1293.

Chester, W. 1960 Adv. Appl. Mech. 6, 120.

Chisneld, R. F. 1957 J. Fluid Mech. 2, 286.

GUDERLEX, G. 1942 Luftfahrtforschung, 19, 302.

LAU, J. 1971 C.A.S.I. Trans. 4, 13.

McEwan, A. D. 1968 Australian Def. Sci. Serv. Aero. Note, no. 298.

Milton, B. E. \& ARCheR, E. D. 1969 A.I.A.A. J. 7, 779.

Russell, D. A. 1967 J. Fluid Mech. 27, 305.

Setcheld, R. E. 1971 Ph.D. thesis, California Institute of Technology.

Skews, B. W. 1972 A.I.A.A. J. 10, 839.

Sмiтh, J. A. 1967 Ph.D. thesis, California Institute of Technology.

Whiтhaм, G. B. 1957 J. Fluid Mech. 2, 145.

Whithaм, G. B. 1958 J. Fluid Mech. 4, 337.

Whiтнам, G. B. 1959 J. Fluid Mech. 5, 369.

+ The initial rise displayed by two of the curves results from starting the reflected shock close to the sonic point. 